\title{
Evidence Based Public Health Practice: A Fundamental Concept
}

\author{
Rajan Paudel \\ Department of Community Medicine and Family Health \\ Institute of Medicine, Kathmandu
}

\section{Introduction}

Evidence based Public Health (EBPH) is the process of refining and disseminating the best available evidence (whether from research, practice or experience) and using that evidence to inform and improve public health policy and practice. Simply it is finding, using and sharing what works in public health. Evidence based Public Health acknowledges the many factors, beyond simply the evidence, that influence decision-making. EBPH is a complex, multi-disciplinary process that occurs within dynamic and ever-changing communities and encompasses different sectors of society. Effective evaluation of evidence to support public health practice must address these multidimensional issues and adequately incorporate community perspectives and values. ${ }^{1}$

Evidence-based public health (EBPH) has been proposed as a practice model that builds upon the success of evidencebased medicine (EBM). EBM has been described as a more scientific and systematic approach to the practice of medicine. It has enhanced medical training and practice in many settings. ${ }^{2}$ Evidence-based Public Health depends on good sound evidence. Although decisions to develop and implement new programs and services must be grounded in best practices, the methods and frameworks needed to inform knowledge and translate evidence into practice are often considered time-consuming and difficult to understand. Public health professionals live in a world of heavy workloads, inadequate staffing and insufficient dedicated resources. Especially in the face these realities, critical appraisal of existing evidence is fundamental to the search for quality evidence to inform the process of public health decision-making. ${ }^{3}$

There are two aspects of evidence based public health: (1) the use of a particular type of evidence to inform public health decisions; and (2) an emphasis on clear reasoning in the process of appraising and interpreting that evidence. The types of research that are commonly associated with evidence based public health are described below. ${ }^{4}$

$\$$ Descriptive: to identify the qualities and distributions of variables;

$\$$ Taxonomic: to compare and classify variables into related groups or categories;

$\$$ Analytic: to examine associations between variables - these may be hypothesised causal or therapeutic relations;
\$ Interpretive: to identify and explain meanings, usually from a particular perspective;

\$ Explanatory: to make observations intelligible and understandable; and

$\$$ Evaluative: to determine quality and worth-often assessing the relevance, effectiveness, and consequences of activities.

\section{Evolution of Evidence-based Public Health}

Formal discourse on the nature and scope of EBPH originated about a decade ago. Several authors have attempted to define EBPH. In 1997, Jenicek defined EBPH as the "conscientious, explicit, and judicious use of current best evidence in making decisions about the care of communities and populations in the domain of health protection, disease prevention, health maintenance and improvement (health promotion)". ${ }^{5}$ In 1999, scholars and practitioners in Australia and the United States elaborated further on the concept of EBPH. Glasziou and colleagues posed a series of questions to enhance uptake of EBPH (e.g., "Does this intervention help alleviate this problem?") and identified 14 sources of high-quality evidence. ${ }^{6}$ In 2004 , Rychetnik and colleagues summarized many key concepts in a glossary for EBPH. ${ }^{7}$

\section{Domains of Evidence-based Public Health Practice}

A consensus has been developed among investigators and public health leaders that a combination of scientific evidence and values, resources, and context should enter into decision making ${ }^{8}$ (fig. 1). In summarizing these various attributes of EBPH, key characteristics include 9 ;

$\$$ Making decisions using the best available peerreviewed evidence (both quantitative and qualitative research)

\$ Using data and information systems systematically,

$\$$ Applying program-planning frameworks (that often have a foundation in behavioral

$\$$ science theory),

$\S$ Engaging the community in assessment and decision making,

\$ Conducting sound evaluation, and

$\$$ Disseminating what is learned to key stakeholders and decision makers. Accomplishing these activities in EBPH is likely to require a synthesis of scientific skills, enhanced communication, common sense, and political acumen. 


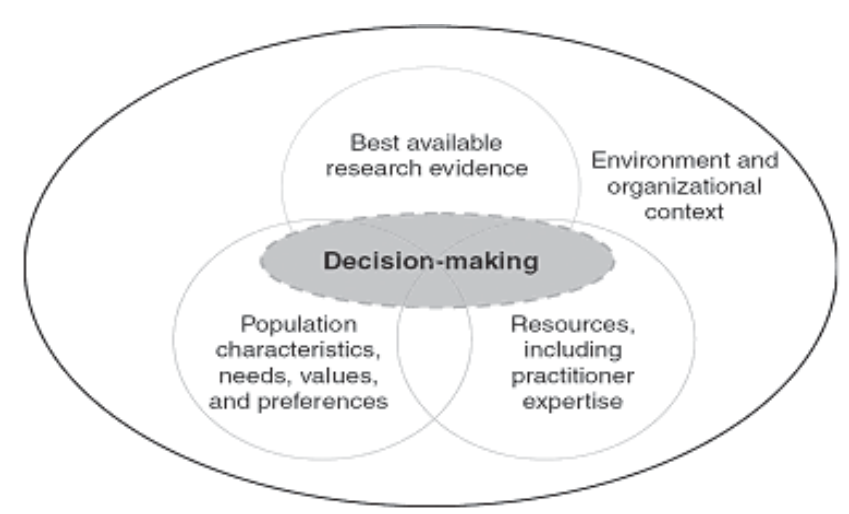

Fig 1: Domains that influence evidence-based decision making

\section{Basic Principals of Public Health Practice}

Public health has been grounded in the science of epidemiology with an emphasis on quantitative methods to address questions of prevalence, effectiveness, and causation. Within this model, health outcomes are primarily attributed to physical, psychological, biological, or environmental causes with interventions focused on encouraging changes in the behavior of individuals. Increasingly, there is acknowledgement of the importance of the social and physical environment determinants of health and the need to understand how contextual factors influence behavior and experience. A greater depth of understanding is needed than quantitative methods supply. Quantitative methods answer questions of effectiveness and causation. Qualitative methods answer questions of how and why. Using a combination of research methods may be the best approach for public health related research that evaluates interventions or strategies..$^{10,11}$

For some public health concerns, a sufficient body of research-based knowledge has not yet accrued. In other situations, the appropriate action is based on a different rationale. For example, science does not drive ethical decision-making though it may provide helpful insights. Likewise, the practice of public health is often determined by regulations, laws, and public policies rather than purely scientific rationale. ${ }^{12}$

\section{Conclusion}

The successful implementation of EBPH in public health practice is both a science and an art. The science is built on epidemiologic, behavioral, and policy research showing the size and scope of a public health problem and which interventions are likely to be effective in addressing the problem. The art of decision making often involves knowing which information is important to a particular stakeholder at the right time. Unlike solving a math problem, significant decisions in public health must balance science and art because rational, evidence-based decision making often involves choosing one alternative from among a set of rational choices. Numerous benefits accrue when decisions in public health are based on sound scientific evidence. While evidence should be weighed along with community beliefs, opinions, and local considerations, evidence-based recommendations are very useful for formulating choices among programs likely to be effective and avoiding ineffective or even harmful interventions. By applying the concepts of EBPH, decision making and, ultimately, public health practice can be improved.

\section{References}

1. NCCMT. Fact Sheet, Strong Evidence, Strong Public Health. National Collaborating Centre for Methods and Tools. Accessed in 18 August 2011. Available From http://www.nccph.ca.

2. Kohatsu ND, Robinson JG, Torner JC. Evidence based Public Health: An Emerging Concept. American Journal of Preventive Medicine; 2004, 27: 417-421.

3. Ciliska D, Thomas H, Buffett C. An Introduction to Evidence-Informed Public Health and A Compendium of Critical Appraisal Tools for Public Health Practice. National Collaborating Centre for Methods and Tools (NCCMT), School of Nursing, McMaster University; Feb 2008.

4. Rychetnik L, Hawe P, Waters E. A glossary for evidence based public health. J. Epidemiol. Community Health; 2004, 8: 538-545

5. Jenicek M. Epidemiology, evidence-based medicine, and evidence-based public health. J. Epidemiol. Commun. Health; 1997, 7:187-97

6. Glasziou P, Longbottom H. Evidence-based public health practice. Aust. N. Z. J. Public Health; 1999, 23:436-40.

7. Rychetnik L, Hawe P, Waters E, Barratt A, Frommer M. A glossary for evidence based public health. J. Epidemiol. Community Health; 2004, 58:538-45

8. Spring B, Walker B, Brownson R, Mullen E, Newhouse $\mathrm{R}$, et al. Definition and competencies for evidencebased behavioral practice. White paper prepared by the Counc. on Evidence-Based Behav. Pract., Northwestern Univ., Chicago, IL; 2008.

9. Brownson RC, Fielding JE, Maylahn CM. EvidenceBased Public Health: A Fundamental Concept for Public Health Practice. Annu. Rev. Public Health; 2009, 30:175-201

10. Fielding JE, \& Briss PA. Promoting evidence-based public health policy: Can we have better evidence and more action? Health Affairs; 2006, 25: 969-978.

11. Jack SM. Utility of qualitative research findings in evidence-based public health practice. Public Health Nursing; 2006, 23: 277-283.

12. Kohatsu, ND, Robinson, JG, \& Torner, JC. Evidencebased public health: An evolving concept. American Journal of Preventive Medicine; 2004, 27: 417-421. 